\title{
ON SUMS OF INDEPENDENT GENERALIZED PARETO RANDOM VARIABLES WITH APPLICATIONS TO INSURANCE AND CAT BONDS
}

DOI:

10.1017/S0269964817000055

\section{Document Version \\ Accepted author manuscript}

Link to publication record in Manchester Research Explorer

Citation for published version (APA):

Nadarajah, S., Zhang, Y., \& Pogány, T. K. (2017). ON SUMS OF INDEPENDENT GENERALIZED PARETO RANDOM VARIABLES WITH APPLICATIONS TO INSURANCE AND CAT BONDS. Probability in the Engineering and Informational Sciences, 1-10. https://doi.org/10.1017/S0269964817000055

\section{Published in:}

Probability in the Engineering and Informational Sciences

\section{Citing this paper}

Please note that where the full-text provided on Manchester Research Explorer is the Author Accepted Manuscript or Proof version this may differ from the final Published version. If citing, it is advised that you check and use the publisher's definitive version.

\section{General rights}

Copyright and moral rights for the publications made accessible in the Research Explorer are retained by the authors and/or other copyright owners and it is a condition of accessing publications that users recognise and abide by the legal requirements associated with these rights.

\section{Takedown policy}

If you believe that this document breaches copyright please refer to the University of Manchester's Takedown Procedures [http://man.ac.uk/04Y6Bo] or contact uml.scholarlycommunications@manchester.ac.uk providing relevant details, so we can investigate your claim.

\section{OPEN ACCESS}




\title{
On sums of independent generalized Pareto random variables with applications to insurance and CAT bonds
}

by

Saralees Nadarajah, School of Mathematics, University of Manchester, Manchester, UK Yuanyuan Zhaang, School of Mathematics, University of Manchester, Manchester, UK Tibor K. Pogány, Faculty of Maritime Studies, University of Rijeka, Rijeka, CROATIA

\begin{abstract}
A longstanding open problem in statistics is: what is the exact distribution of the sum of independent generalized Pareto or Pareto random variables? Here, we derive single integral representations for the exact distribution with the integrand involving the incomplete and complementary incomplete gamma functions. Applications to insurance and catastrophe bonds are described.
\end{abstract}

2000 mathematics subject classification: Primary 60E10; Secondary 33C60, 62G32.

Keywords and phrases: Characteristic function; Generalized Pareto distribution; Incomplete gamma function.

\section{Introduction}

The Pareto distribution due to Pareto (1964) is the most popular statistical model in economics, finance and related areas. A most general form of the Pareto distribution is the generalized Pareto distribution due to Pickands (1975). Its cumulative distribution function (CDF) and probability density function (PDF) are specified by

$$
F(x)=1-\left[1+\frac{\xi(x-\mu)}{\sigma}\right]^{-1 / \xi}
$$

and

$$
f(x)=\frac{1}{\sigma}\left[1+\frac{\xi(x-\mu)}{\sigma}\right]^{-1 / \xi-1},
$$

respectively, for $\mu<x<\infty$ if $\xi \geq 0$ and $\mu<x<\mu-\sigma / \xi$ if $\xi<0$, where $-\infty<\mu<\infty$ is the location parameter, $\sigma>0$ is the scale parameter, and $-\infty<\xi<\infty$ is the shape parameter. The case $\xi=0$ should be interpreted as a limiting case. In this case, (1) and (2) give the exponential distribution with location parameter $-\infty<\mu<\infty$ and scale parameter $\sigma>0$. The case $\xi>0$ gives a heavy tailed distribution. The case $\xi<0$ gives a light tailed distribution.

An outstanding research question in statistics is: what is the exact distribution of the sum of independent generalized Pareto or Pareto random variables? Many authors have attempted to 
answer this question: Hitha (1991, page 9) says "The distribution of a sum of Pareto variables that are independent and identically distributed is difficult to obtain"; Bean (2001, page 235) says "There is no simple relationship for an independent sum of Pareto random variables"; Goovaerts et al. (2005, Theorems 2.1 and 2.2) derive asymptotic forms for the distribution of a sum of Pareto random variables; Zaliapin et al. (2005) present five different approximations for the sum of independent Pareto random variables; Hempel (2007) says "Unfortunately, the performance of this test statistic is impossible to compute analytically and difficult to compute numerically because the exponential terms in the last summation are Pareto distributed. Pareto distributions are very heavy tailed and the distribution of the natural logarithm of a sum of Pareto random variables cannot be derived analytically"; R-forge distributions Core Team (2008) say "The convolution (i.e. sum) of Pareto I distributions does not have any particular form but the product of Pareto I distributions does have an analytical form"; Albrecher and Kortschak (2009, Theorem 2.1) derive an integral representation for the tail of the distribution of a sum of Pareto random variables; Bonfiglioli and Gancia (2013) say that the "sum of Pareto distributions is intractable"; to mention just a few.

Areas where sums of generalized Pareto or Pareto random variables arise include: ruin theory and reinsurance pricing (Morales, 2005); modeling of natural and human-induced processes (Zaliapin et al., 2005); models for significant wave height (Bazargan et al., 2007); track initialization for multi-static active sonar systems (Hempel, 2007); portfolio's aggregate losses and waiting time distributions (Ramsay, 2006, 2007, 2008, 2009).

To the best of our knowledge, the exact distribution of the sum of generalized Pareto or Pareto random variables has not been derived. But we have come to know of a paper by Nguyen and Robert (2013) that is currently under review for publication. A preprint of it is available on-line at http://docs.isfa.fr/labo/2012.16.pdf. The main theorem (Theorem 1) in Nguyen and Robert (2013) gives an expression for the CDF of $X_{1}+X_{2}+\cdots+X_{n}$, a sum of $n$ independent Pareto random variables. The expression involves multiple infinite sums, multiple products and multiple finite sums. In fact, it is easy to see that the expression given by Theorem 1 of Nguyen and Robert (2013) involves

$$
2^{n}+\sum_{i=1}^{2^{n}-2} 2^{\left|\theta_{i, n}\right|+1}
$$

infinite sums, where $\left\{\theta_{1, n}, \theta_{2, n}, \ldots, \theta_{2^{n}-2, n}\right\}$ is the set of all subsets of $\{1,2, \ldots, n\}$, excluding the empty set and the full set. In addition, the expression given by Theorem 1 of Nguyen and Robert (2013) involves at least

$$
1+\sum_{i=1}^{2^{n}-2}\left[1+\sum_{k=0}^{\infty}\left(\begin{array}{c}
k+1 \\
\left|\theta_{i, n}^{c}\right|
\end{array}\right)\right]
$$

products, where $\theta_{i, n}^{c}$ denotes the complement of $\theta_{i, n}$. In addition, the expression given by Theorem 1 of Nguyen and Robert (2013) involves an infinite number of finite sums. Clearly, this is a complicated expression. Its computation will become prohibitive as $n$ becomes large. In this note, we give expressions for the CDF of $X_{1}+X_{2}+\cdots+X_{n}$ taking the form of a single integral, much simpler than the expression in Nguyen and Robert (2013). For generality, we consider generalized Pareto random variables and not Pareto random variables.

One of the tools used to derive the distribution of sums of independent random variables is the characteristic function $(\mathrm{CHF})$. The $\mathrm{CHF}$ of a random variable, $X$ say, defined by $\phi_{X}(t)=$ 
$\mathrm{E} \exp \{\mathrm{it} X\}$, where $\mathrm{i}=\sqrt{-1}$, is a fundamental tool in probability. The CHF can be used to derive the distribution of $X_{1}+X_{2}+\cdots+X_{n}$ when $X_{i}, i=1,2, \ldots, n$ are independent generalized Pareto random variables.

If $X$ is a generalized Pareto random variable with $\xi=0$ then it is well known that

$$
\phi_{X}(t)=\frac{\exp (\mathrm{i} \mu t)}{1-\mathrm{i} \sigma t} .
$$

The result in (3) has been used to derive the distribution of the sum of independent but not necessarily identical exponential random variables (this distribution is in general different from the gamma distribution), see Amari and Misra (1997) and Khuong and Kong (2006).

For generalized Pareto random variables with $\xi \neq 0$, closed form expressions for $\phi_{X}(t)$ have not been known in the literature. Closed form expressions for $\phi_{X}(t)$ for some related random variables, the extreme value random variables, were derived only recently, see Nadarajah and Pogány (2013).

The main results of this note are: explicit closed form expressions for the CHF for the generalized Pareto random variable, a single integral representation for the PDF of the sum of independent generalized Pareto random variables, and a single integral representation for the CDF of the sum of independent generalized Pareto random variables; applications to insurance and catastrophe bonds. The expressions given in Section 2 involve the incomplete and complementary incomplete gamma functions defined by $\gamma(a, z)=\int_{0}^{z} t^{a-1} \exp (-t) d t$ and $\Gamma(a, z)=\int_{z}^{\infty} t^{a-1} \exp (-t) d t$, respectively.

Incomplete gamma functions are included as in-built functions in most mathematical software packages, so they can be easily evaluated by the software packages Maple, Matlab and Mathematica using known procedures. Using these in-built functions, Section 3 describes an application to insurance of the results in Section 2. Section 4 describes an application to catastrophe bonds.

\section{Main results}

Our main results are Theorem 2.1, Theorem 2.2 and Theorem 2.3. Theorem 2.1 derives an explicit closed form expression for the CHF for the generalized Pareto random variable. Theorem 2.2 derives a single integral representation for the PDF of the sum of independent generalized Pareto random variables. Theorem 2.3 derives a single integral representation for the CDF of the sum of independent generalized Pareto random variables.

Theorem 2.1 Let $X$ denote the generalized Pareto random variable. Its $\mathrm{CHF}$ has the closed form:

$$
\phi_{X}(t)= \begin{cases}-\xi^{-1 / \xi-1}(t \sigma)^{1 / \xi} \exp \{\mathrm{i} t(\mu-\sigma / \xi)-\mathrm{i} \pi /(2 \xi)\} \gamma(-1 / \xi,-\mathrm{i} t \sigma / \xi), & \text { if } \xi<0, \\ \xi^{-1 / \xi-1}(t \sigma)^{1 / \xi} \exp \{\mathrm{i} t(\mu-\sigma / \xi)-\mathrm{i} \pi /(2 \xi)\} \Gamma(-1 / \xi,-\mathrm{i} t \sigma / \xi), & \text { if } \xi>0\end{cases}
$$

for all $-\infty<\mu<\infty$ and $\sigma>0$. The CHF for $\xi=0$ is given by (3). 
Proof: Consider first the case $\xi<0$. Then,

$$
\begin{aligned}
\phi_{X}(t) & =\frac{1}{\sigma} \int_{\mu}^{\mu-\sigma / \xi} \exp (\mathrm{i} t x)\left[1+\frac{\xi(x-\mu)}{\sigma}\right]^{-1 / \xi-1} d x \\
& =-\xi^{-1} \exp \{\mathrm{i} t(\mu-\sigma / \xi)\} \int_{0}^{1} \exp \{-(-\mathrm{i} t \sigma / \xi) y\} y^{-1 / \xi-1} d y
\end{aligned}
$$

The result follows from the definition of the incomplete gamma function.

Consider now the case $\xi>0$. Then,

$$
\begin{aligned}
\phi_{X}(t) & =\frac{1}{\sigma} \int_{\mu}^{\infty} \exp (\mathrm{i} t x)\left[1+\frac{\xi(x-\mu)}{\sigma}\right]^{-1 / \xi-1} d x \\
& =\xi^{-1} \exp \{\mathrm{i} t(\mu-\sigma / \xi)\} \int_{1}^{\infty} \exp \{-(-\mathrm{i} t \sigma / \xi) y\} y^{-1 / \xi-1} d y .
\end{aligned}
$$

The result follows from the definition of the complementary incomplete gamma function.

Theorem 2.2 Suppose $X_{i}, i=1,2, \ldots, N$ are independent generalized Pareto random variables with parameters $\left(\mu_{i}, \sigma_{i}, \xi_{i}\right)$. The PDF of $Z=X_{1}+X_{2}+\cdots+X_{N}$ for fixed $N$ can be expressed as

$$
\begin{aligned}
f_{Z}(z)= & \frac{1}{2 \pi} \int_{-\infty}^{\infty} \exp \left(\mathrm{i} t \sum_{i=1}^{N} \mu_{i}-\mathrm{i} z t\right) \\
& \cdot \prod_{\xi_{i}<0}\left[-\xi_{i}^{-1 / \xi_{i}-1}\left(t \sigma_{i}\right)^{1 / \xi_{i}} \exp \left\{-\mathrm{i} t \sigma_{i} / \xi_{i}-\mathrm{i} \pi /\left(2 \xi_{i}\right)\right\} \gamma\left(-1 / \xi_{i},-\mathrm{i} t \sigma_{i} / \xi_{i}\right)\right] \\
& \cdot \prod_{\xi_{i}>0}\left[\xi_{i}^{-1 / \xi_{i}-1}\left(t \sigma_{i}\right)^{1 / \xi_{i}} \exp \left\{-\mathrm{i} t \sigma_{i} / \xi_{i}-\mathrm{i} \pi /\left(2 \xi_{i}\right)\right\} \Gamma\left(-1 / \xi_{i},-\mathrm{i} t \sigma_{i} / \xi_{i}\right)\right] \\
& \cdot \prod_{\xi_{i}=0}\left(1-\mathrm{i} \sigma_{i} t\right)^{-1} d t
\end{aligned}
$$

for $-\infty<z<\infty$.

Proof: Follows by using Theorem 2.1 and the inversion theorem.

Theorem 2.3 Suppose $X_{i}, i=1,2, \ldots, N$ are independent generalized Pareto random variables with parameters $\left(\mu_{i}, \sigma_{i}, \xi_{i}\right)$. The CDF of $Z=X_{1}+X_{2}+\cdots+X_{N}$ for fixed $N$ can be expressed as

$$
\begin{aligned}
F_{Z}(z)= & \frac{1}{2}-\frac{1}{\pi} \int_{0}^{\infty} t^{-1} \operatorname{Im}\left[\exp \left(\mathrm{i} t \sum_{i=1}^{N} \mu_{i}-\mathrm{i} z t\right)\right. \\
& \cdot \prod_{\xi_{i}<0}\left[-\xi_{i}^{-1 / \xi_{i}-1}\left(t \sigma_{i}\right)^{1 / \xi_{i}} \exp \left\{-\mathrm{i} t \sigma_{i} / \xi_{i}-\mathrm{i} \pi /\left(2 \xi_{i}\right)\right\} \gamma\left(-1 / \xi_{i},-\mathrm{i} t \sigma_{i} / \xi_{i}\right)\right] \\
& \cdot \prod_{\xi_{i}>0}\left[\xi_{i}^{-1 / \xi_{i}-1}\left(t \sigma_{i}\right)^{1 / \xi_{i}} \exp \left\{-\mathrm{i} t \sigma_{i} / \xi_{i}-\mathrm{i} \pi /\left(2 \xi_{i}\right)\right\} \Gamma\left(-1 / \xi_{i},-\mathrm{i} t \sigma_{i} / \xi_{i}\right)\right] \\
& \left.\cdot \prod_{\xi_{i}=0}\left(1-\mathrm{i} \sigma_{i} t\right)^{-1}\right] d t
\end{aligned}
$$

for $-\infty<z<\infty$, where $\operatorname{Im}(\cdot)$ denotes the imaginary part. 
Proof: Follows by using Theorem 2.1 and the inversion theorem of Wendel (1961).

There is a variety of other inversion formulas than those used in Theorems 2.2 and 2.3. See Abate and Valkó (2004), Ramsay (2006, 2008) and Albrecher and Kortschak (2009). Of these the first three have the advantage that the absolute value of the integral is integrable. The last one is only an approximation.

The integrals in (4)-(5) do not appear to have closed forms. However, they can be easily computed using known routines for incomplete and complementary incomplete gamma functions.

Theorems 2.2 and 2.3 can be easily extended to the case that $N$ is a discrete random variable. In this case, the PDF and the CDF of $Z$ will take the form of a single summation with each term involving a single integral.

Corollaries 2.1 and 2.2 are particular cases of Theorem 2.1. Corollary 2.1 considers the particular case that $1 / \xi$ is a positive integer or a negative integer. When $1 / \xi$ is a negative integer $\phi_{X}(t)$ is reduced to an elementary form. When $1 / \xi$ is a positive integer $\phi_{X}(t)$ is reduced to an elementary form except for $\operatorname{Ei}(\cdot)$, the exponential integral defined by $\operatorname{Ei}(x)=\int_{-\infty}^{x} \exp (t) / t d t$.

Corollary 2.2 considers the particular case that $1 / \xi$ is a half positive integer or a half negative integer. In these cases, $\phi_{X}(t)$ is reduced to elementary forms except for $\Phi(\cdot)$, the CDF of a standard normal random variable. In-built routines for $\operatorname{Ei}(\cdot)$ and $\Phi(\cdot)$ are widely available, even in pocket calculators.

Corollary 2.1 Let $X$ denote the generalized Pareto random variable with $1 / \xi$ an integer. Its $\mathrm{CHF}$ has the closed form:

$$
\phi_{X}(t)=\left\{\begin{array}{l}
-(-1 / \xi-1) ! \xi^{-1 / \xi-1}(t \sigma)^{1 / \xi} \exp \{\mathrm{i} t(\mu-\sigma / \xi)-\mathrm{i} \pi /(2 \xi)\} \\
+(-1 / \xi-1) ! \xi^{-1 / \xi-1}(t \sigma)^{1 / \xi} \exp \{\mathrm{i} t \mu-\mathrm{i} \pi /(2 \xi)\} \sum_{k=0}^{-1 / \xi-1} \frac{z^{k}}{k !}, \\
\text { if } \xi<0, \\
\frac{(-1)^{1 / \xi-1}}{(1 / \xi) !} \xi^{-1 / \xi-1}(t \sigma)^{1 / \xi} \exp \{\mathrm{i} t(\mu-\sigma / \xi)-\mathrm{i} \pi /(2 \xi)\} \operatorname{Ei}(-z) \\
+\frac{(-1)^{1 / \xi-1}}{(1 / \xi) !} \xi^{-1 / \xi-1}(t \sigma)^{1 / \xi} \exp \{\mathrm{i} t(\mu-\sigma / \xi)-\mathrm{i} \pi /(2 \xi)\} \log z \\
-\frac{(-1)^{1 / \xi-1}}{2(1 / \xi) !} \xi^{-1 / \xi-1}(t \sigma)^{1 / \xi} \exp \{\mathrm{i} t(\mu-\sigma / \xi)-\mathrm{i} \pi /(2 \xi)\} \log (-z) \\
+\frac{(-1)^{1 / \xi-1}}{2(1 / \xi) !} \xi^{-1 / \xi-1}(t \sigma)^{1 / \xi} \exp \{\mathrm{i} t(\mu-\sigma / \xi)-\mathrm{i} \pi /(2 \xi)\} \log \left(-\frac{1}{z}\right) \\
-\xi^{-1 / \xi-1}(t \sigma)^{1 / \xi} \exp \{\mathrm{i} t \mu-\mathrm{i} \pi /(2 \xi)\} \sum_{k=1}^{1 / \xi} \frac{z^{k-1 / \xi-1}}{(-1 / \xi)_{k}}, \\
\text { if } \xi>0,
\end{array}\right.
$$

where $z=-\mathrm{i} t \sigma / \xi$ and $(e)_{k}=e(e+1) \cdots(e+k-1)$ denotes the ascending factorial.

Proof: Follows by using http: // functions. wolfram. com/06.06.03.0009.01 and http: // functions. wolfram. com/06.06.03.0011.01 to simplify $\gamma(-1 / \xi,-i t \sigma / \xi)=\Gamma(-1 / \xi)-\Gamma(-1 / \xi,-i t \sigma / \xi)$ and 
$\Gamma(-1 / \xi,-\mathrm{i} t \sigma / \xi)$, respectively.

Corollary 2.2 Let $X$ denote the generalized Pareto random variable with $1 / \xi$ a half integer. Its $\mathrm{CHF}$ has the closed form:

$$
\phi_{X}(t)=\left\{\begin{array}{l}
\xi^{-1 / \xi-1}(t \sigma)^{1 / \xi} \exp \{\mathrm{i} t(\mu-\sigma / \xi)-\mathrm{i} \pi /(2 \xi)\} \Gamma(-1 / \xi) \\
-2 \xi^{-1 / \xi-1}(t \sigma)^{1 / \xi} \exp \{\mathrm{i} t(\mu-\sigma / \xi)-\mathrm{i} \pi /(2 \xi)\} \Gamma(-1 / \xi) \Phi(\sqrt{2 z}) \\
+(-1)^{-1 / \xi-3 / 2} \xi^{-1 / \xi-1}(t \sigma)^{1 / \xi} \exp \{\mathrm{i} t \mu-\mathrm{i} \pi /(2 \xi)\} \sqrt{z} \sum_{k=0}^{-1 / \xi-3 / 2}\left(1+\frac{1}{\xi}\right)_{-1 / \xi-3 / 2-k} \\
\text { if } \xi<0, \\
\frac{2 \sqrt{\pi}(-1)^{1 / 2+1 / \xi}}{(1 / 2)_{1 / 2+1 / \xi}} \xi^{-1 / \xi-1}(t \sigma)^{1 / \xi} \exp \{\mathrm{i} t(\mu-\sigma / \xi)-\mathrm{i} \pi /(2 \xi)\} \\
-\frac{2 \sqrt{\pi}(-1)^{1 / 2+1 / \xi}}{(1 / 2)_{1 / 2+1 / \xi}} \xi^{-1 / \xi-1}(t \sigma)^{1 / \xi} \exp \{\mathrm{i} t(\mu-\sigma / \xi)-\mathrm{i} \pi /(2 \xi)\} \Phi(\sqrt{2 z}) \\
-\xi^{-1 / \xi-1}(t \sigma)^{1 / \xi} \exp \{\mathrm{i} t \mu-\mathrm{i} \pi /(2 \xi)\} z^{-1 / \xi} \sum_{k=0}^{1 / \xi-1 / 2} \frac{z^{k}}{(-1 / \xi)_{k+1}} \\
\text { if } \xi>0,
\end{array}\right.
$$

where $z=-\mathrm{i} t \sigma / \xi$ and $(e)_{k}=e(e+1) \cdots(e+k-1)$ denotes the ascending factorial.

Proof: Follows by using http: // functions. wolfram. com/06.06.03.0005.01 and http: // functions. wolfram. com/06.06.03.0007.01 to simplify $\gamma(-1 / \xi,-i t \sigma / \xi)=\Gamma(-1 / \xi)-\Gamma(-1 / \xi,-i t \sigma / \xi)$ and $\Gamma(-1 / \xi,-\mathrm{i} t \sigma / \xi)$, respectively.

\section{Application to insurance}

A probability of importance associated with the sum of independent generalized Pareto or Pareto random variables is

$$
P=\operatorname{Pr}\left(X_{1}+X_{2}+\cdots+X_{N}>u\right)
$$

where $N$ could be deterministic or stochastic. For example, (6) could represent the probability that the total claim amount over some period exceeding a certain threshold (Klugman et al., 2008).

The probability, $P$, in (6) follows from (5). If $X_{i}$ are independent generalized Pareto random 
variables with parameters $\left(\mu_{i}, \sigma_{i}, \xi_{i}\right)$ then

$$
\begin{aligned}
P= & \frac{1}{2}+\frac{1}{\pi} \int_{0}^{\infty} t^{-1} \operatorname{Im}\left[\exp \left(\mathrm{i} t \sum_{i=1}^{N} \mu_{i}-\mathrm{i} u t\right)\right. \\
& \cdot \prod_{\xi_{i}<0}\left[-\xi_{i}^{-1 / \xi_{i}-1}\left(t \sigma_{i}\right)^{1 / \xi_{i}} \exp \left\{-\mathrm{i} t \sigma_{i} / \xi_{i}-\mathrm{i} \pi /\left(2 \xi_{i}\right)\right\} \gamma\left(-1 / \xi_{i},-\mathrm{i} t \sigma_{i} / \xi_{i}\right)\right] \\
& \cdot \prod_{\xi_{i}>0}\left[\xi_{i}^{-1 / \xi_{i}-1}\left(t \sigma_{i}\right)^{1 / \xi_{i}} \exp \left\{-\mathrm{i} t \sigma_{i} / \xi_{i}-\mathrm{i} \pi /\left(2 \xi_{i}\right)\right\} \Gamma\left(-1 / \xi_{i},-\mathrm{i} t \sigma_{i} / \xi_{i}\right)\right] \\
& \left.\cdot \prod_{\xi_{i}=0}\left(1-\mathrm{i} \sigma_{i} t\right)^{-1}\right] d t .
\end{aligned}
$$

Note that (7) is a single integral of known special functions. This representation is perhaps the simplest means to compute $P$. The probability, $P$, can also be computed in other ways. For example, it can be computed by using the PDF of generalized Pareto random variables. But $P$ will then be an $(N-1)$-fold integral, a much more complicated representation than (7). Nadarajah (2008) derives explicit expressions (involving the Appell function of the first kind and the Gauss hypergeometric function) for $P$ using this approach when $N=2$.

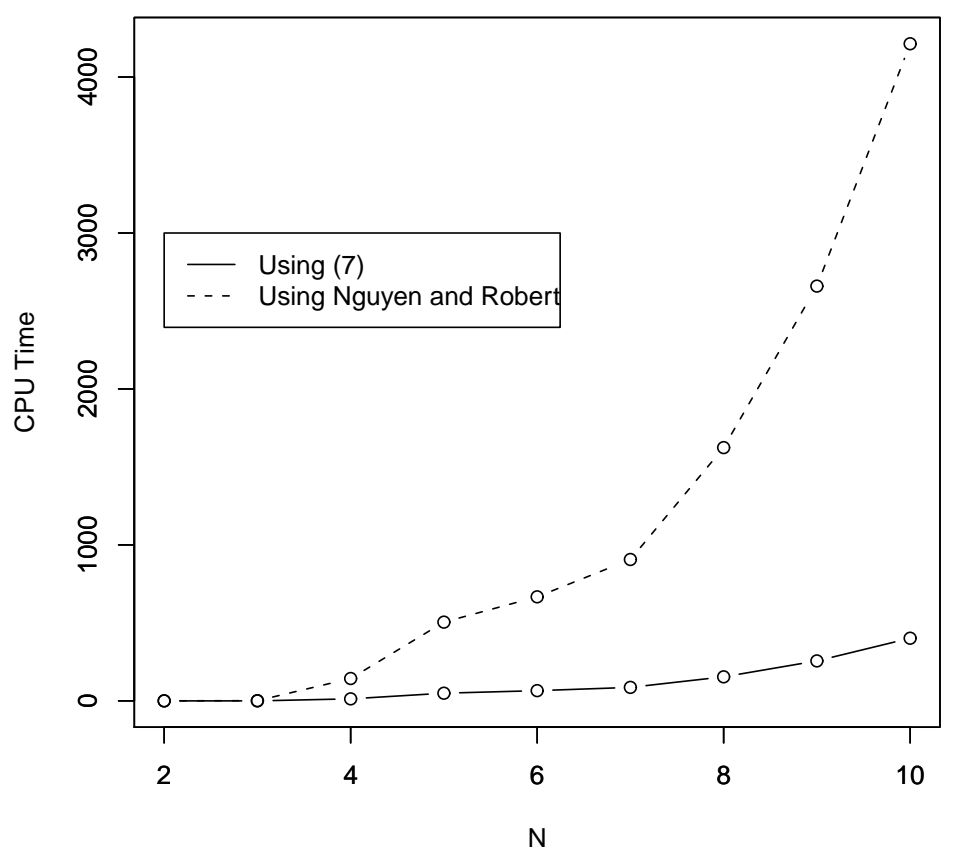

Figure 1: CPU times taken to compute (7) and the corresponding in Nguyen and Robert (2013)'s method versus $N$ when $u=1, \mu_{i}=0, \sigma_{i}=1$ and $\xi_{i}=0.3$. 


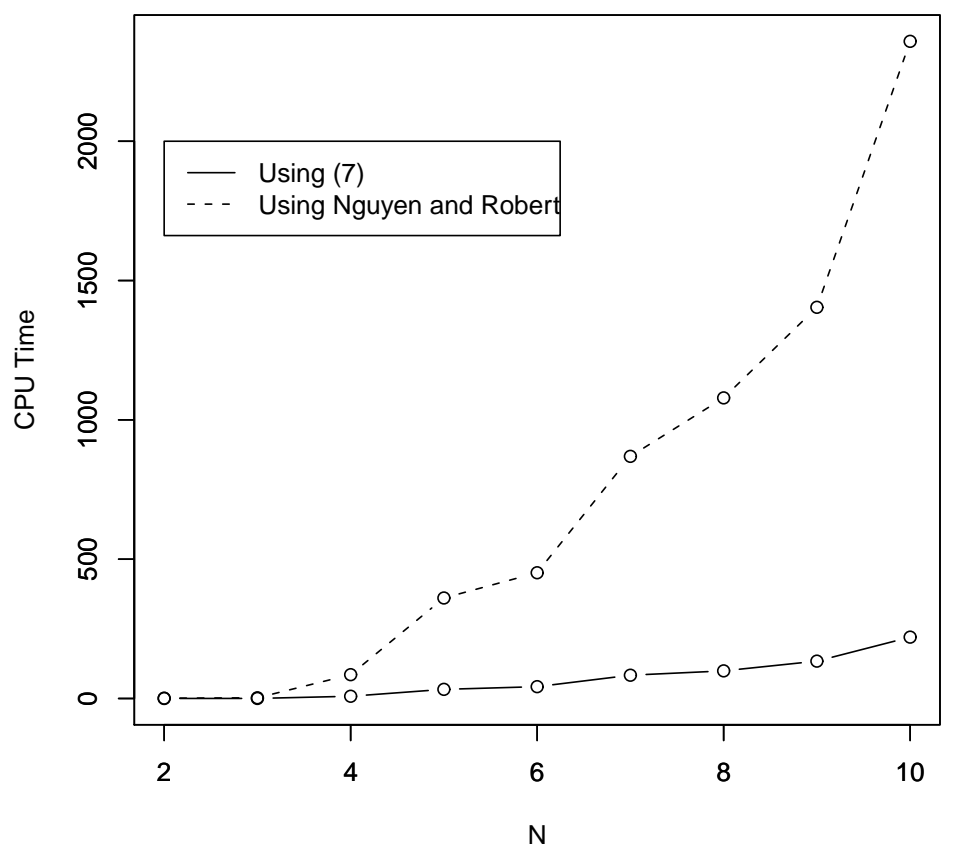

Figure 2: CPU times taken to compute (7) and the corresponding in Nguyen and Robert (2013)'s method versus $N$ when $u=1, \mu_{i}=0, \sigma_{i}=1$ and $\xi_{i}=-0.2$.

We now present some numerical results with respect to computing (7). Figures 1 and 2 show the Central Processing Unit (CPU) time in seconds taken to compute (7) for $u=1, \mu_{i}=0, \sigma_{i}=1$, $\xi_{i}=0.3$ and $u=1, \mu_{i}=0, \sigma_{i}=1, \xi_{i}=-0.2$. The figures show how the time varies with respect to $N$. The figures also show the CPU times taken to compute $P$ by using the expansions in Nguyen and Robert (2013). The CPU times for (7) appear much smaller for every $N$. The CPU time increases with $N$. The increase appears sharp. However, it is comforting to note that the CPU times for (7) are manageable even for $N$ as large as ten.

The computations for Figures 1 and 2 were performed using Mathematica. The accuracy of computations of (7) is not an issue as Mathematica (like most other algebraic manipulation packages) allows for arbitrary precision.

\section{Application to catastrophe bonds}

Catastrophe risk bonds, also known as CAT bonds, are a specific insurance product that creates risk-linked securities which transfer a specific set of risks commonly through catastrophe and natural disaster risks from an issuer or sponsor (insurance or reinsurance companies) to the investors. In this scenario, the investors take on the liability of a specified catastrophe or event occurring in return for an attractive rate of investment. However, in the event of a catastrophe or an extreme event occurring, the investor will forgo the principal that they invested and the issuer will receive 
this money to cover their losses.

The data represents the value of monthly catastrophe bond deal (in millions of dollars) executed from December 1996 to November 2016. The data were obtained from the Artemis Catastrophe Bond deal directory, see http://www.artemis.bm/. The catastrophe bonds were assigned into two broad location categories, these are the USA, and Asia / Europe. Within the two categories, Catastrophe bonds were further sorted into three types of catastrophe groups. These catastrophe groups are earthquakes, wind storms (hurricane, typhoon and cyclone) and other catastrophe (property catastrophe, mortgage, life insurance and medical benefit claims levels).

\begin{tabular}{lllllll}
\hline & \multicolumn{3}{c}{ USA } & \multicolumn{3}{c}{ Asia / Europe } \\
\cline { 2 - 6 } & Earthquakes & Wind storms & Others & Earthquakes & Wind storms & Others \\
\hline Minimum & 3.75 & 7.32 & 25.00 & 3.75 & 43.00 & 47.67 \\
Q1 & 38.00 & 75.00 & 135.00 & 65.22 & 91.75 & 100.00 \\
Median & 150.00 & 187.50 & 234.00 & 150.00 & 108.00 & 130.00 \\
Mean & 176.00 & 215.40 & 238.00 & 188.30 & 150.90 & 170.80 \\
Q3 & 259.00 & 300.00 & 300.00 & 265.20 & 200.00 & 235.00 \\
Maximum & 1100.00 & 1500.00 & 750.00 & 1100.00 & 400.00 & 700.00 \\
Variance & 29258.75 & 44148.33 & 20473.05 & 33355.73 & 7579.681 & 13021.26 \\
Skewness & 2.274 & 3.086 & 0.891 & 2.633 & 1.205 & 2.044 \\
Kurtosis & 11.883 & 17.961 & 4.100 & 13.015 & 3.566 & 9.455 \\
CV & 0.972 & 0.976 & 0.601 & 0.970 & 0.577 & 0.668 \\
\hline
\end{tabular}

Table 1: Some summary statistics of the data.

The following summary statistics of the data are given in Table 1: minimum, first quartile (Q1), median, mean, third quartile (Q3), maximum, variance, skewness, kurtosis and coefficient of variation $(\mathrm{CV})$. The data are generally positively skewed and have peakedness greater than that of the normal distribution. For the USA, the minimum, first quartile, median and mean are largest for other catastrophe while the third quartile, maximum, variance, skewness, kurtosis and coefficient of variation are largest for wind storms. For Asia and Europe, the minimum and first quartile are largest for other catastrophe while the median, third quartile, maximum, variance, skewness, kurtosis and coefficient of variation are largest for earthquakes.

We are interested in determining the distributions of the total catastrophe bond due to earthquakes, the total catastrophe bond due to wind storms and the total catastrophe bond due to other disasters. The total catastrophe bond due to earthquakes are the sum of the catastrophe bonds for earthquakes in the USA and earthquakes in Asia and Europe. Similarly, the total catastrophe bond due to wind storms are the sum of the catastrophe bonds for wind storms in the USA and wind storms in Asia and Europe. Similarly, the total catastrophe bond due to other disasters are the sum of the catastrophe bonds for other disasters in the USA and other disasters in Asia and Europe. 

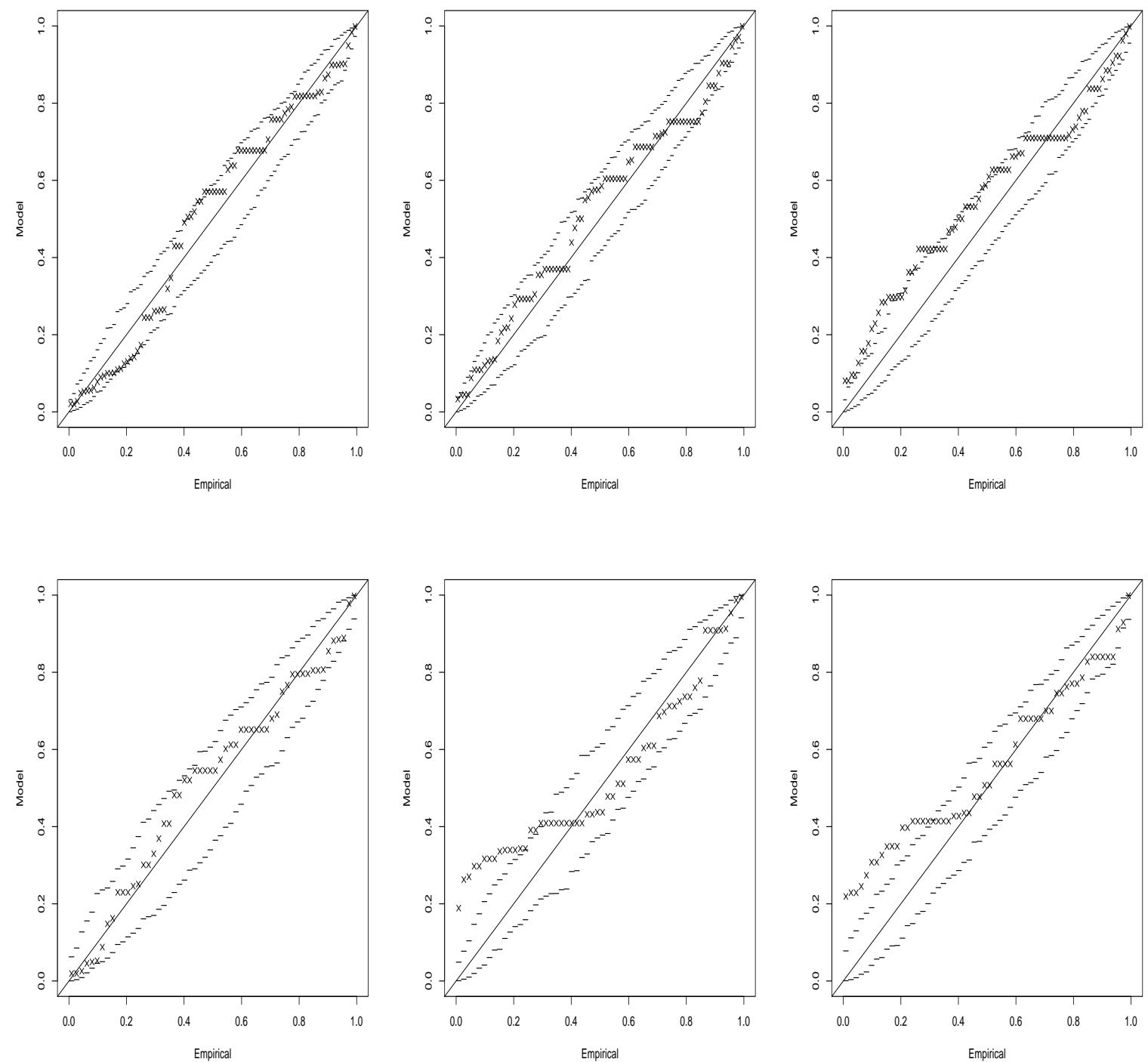

Figure 3: Probability plots of the fit of the generalized Pareto distribution to the catastrophe bond data for earthquakes in the USA (top left), wind storms in the USA (top middle), other disasters in the USA (top right), earthquakes in Asia and Europe (bottom left), wind storms in Asia and Europe (bottom middle) and other disasters in Asia and Europe (bottom right). 

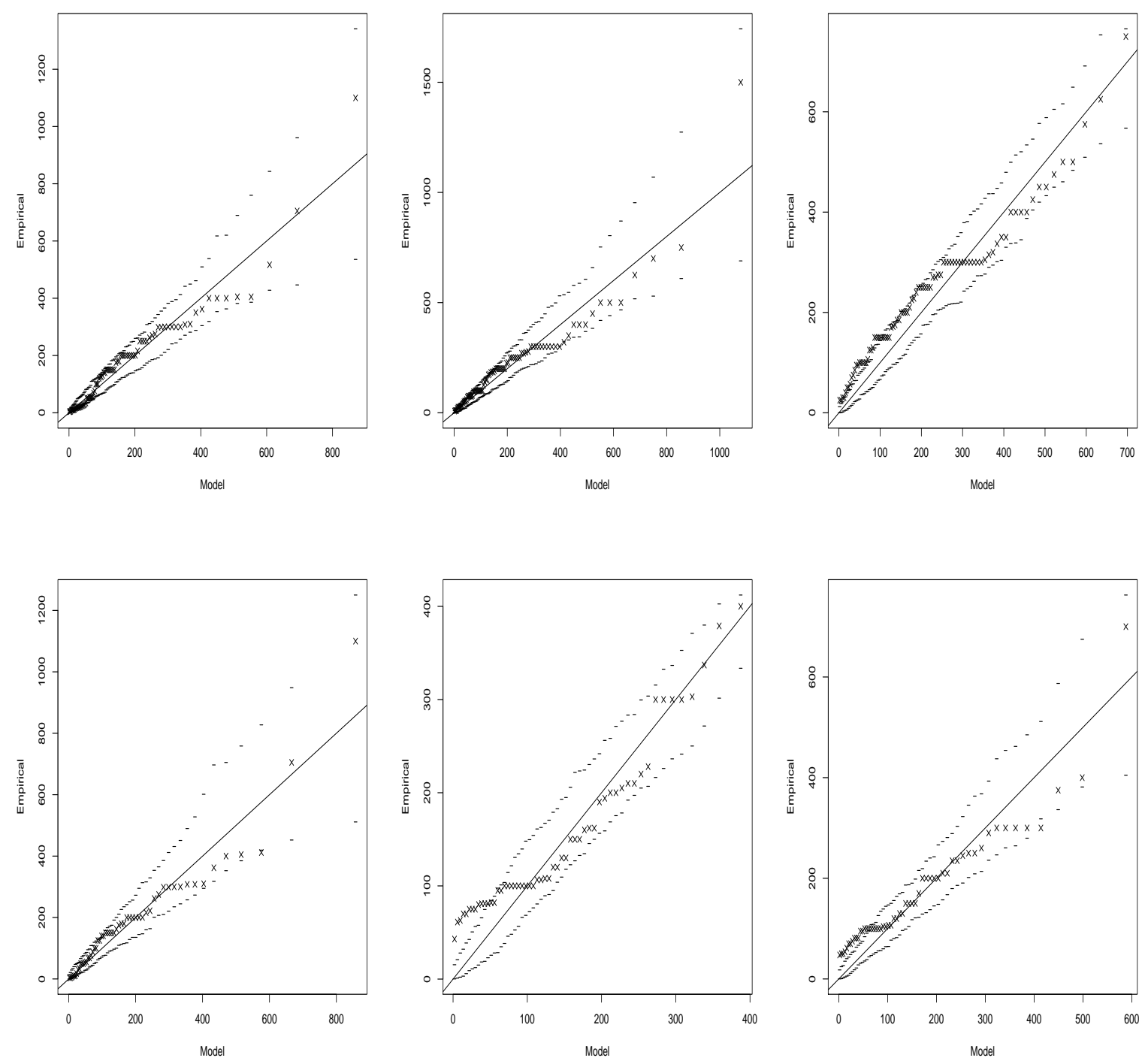

Figure 4: Quantile plots of the fit of the generalized Pareto distribution to the catastrophe bond data for earthquakes in the USA (top left), wind storms in the USA (top middle), other disasters in the USA (top right), earthquakes in Asia and Europe (bottom left), wind storms in Asia and Europe (bottom middle) and other disasters in Asia and Europe (bottom right).

The results in Section 2 can be used to estimate the distributions of the total catastrophe bond. We fitted the generalized Pareto distribution to each of the six data sets. The method of maximum likelihood was used. The parameter estimates and standard errors are given in Table 2 . The standard errors were obtained by inverting the observed information matrix.

The goodness of fit of the generalized Pareto distribution is assessed by the probability and quantile plots shown in Figures 3 and 4. Also shown in these figures are simulated 95 percent confidence intervals. The plots appear reasonable, showing that the fits are reasonable. 


\begin{tabular}{lll}
\hline & USA & Asia / Europe \\
\hline Earthquakes & $\widehat{\sigma_{1}}=179.130(25.119)$, & $\widehat{\sigma_{2}}=192.119(32.487)$, \\
& $\widehat{\xi_{1}}=-0.023(0.091)$ & $\widehat{\xi_{2}}=-0.024(0.105)$ \\
Wind storms & $\widehat{\sigma_{1}}=217.204(28.749)$, & $\widehat{\sigma_{2}}=216.676(32.995)$, \\
& $\widehat{\xi_{1}}=-0.014(0.078)$ & $\widehat{\xi_{2}}=-0.508(0.104)$ \\
Others & $\widehat{\sigma_{1}}=302.204(34.239)$, & $\widehat{\sigma_{2}}=197.92(29.030)$, \\
& $\widehat{\xi_{1}}=-0.369(0.062)$ & $\widehat{\xi_{2}}=-0.214(0.074)$ \\
\hline
\end{tabular}

Table 2: Fitted estimates of the generalized Pareto distribution.
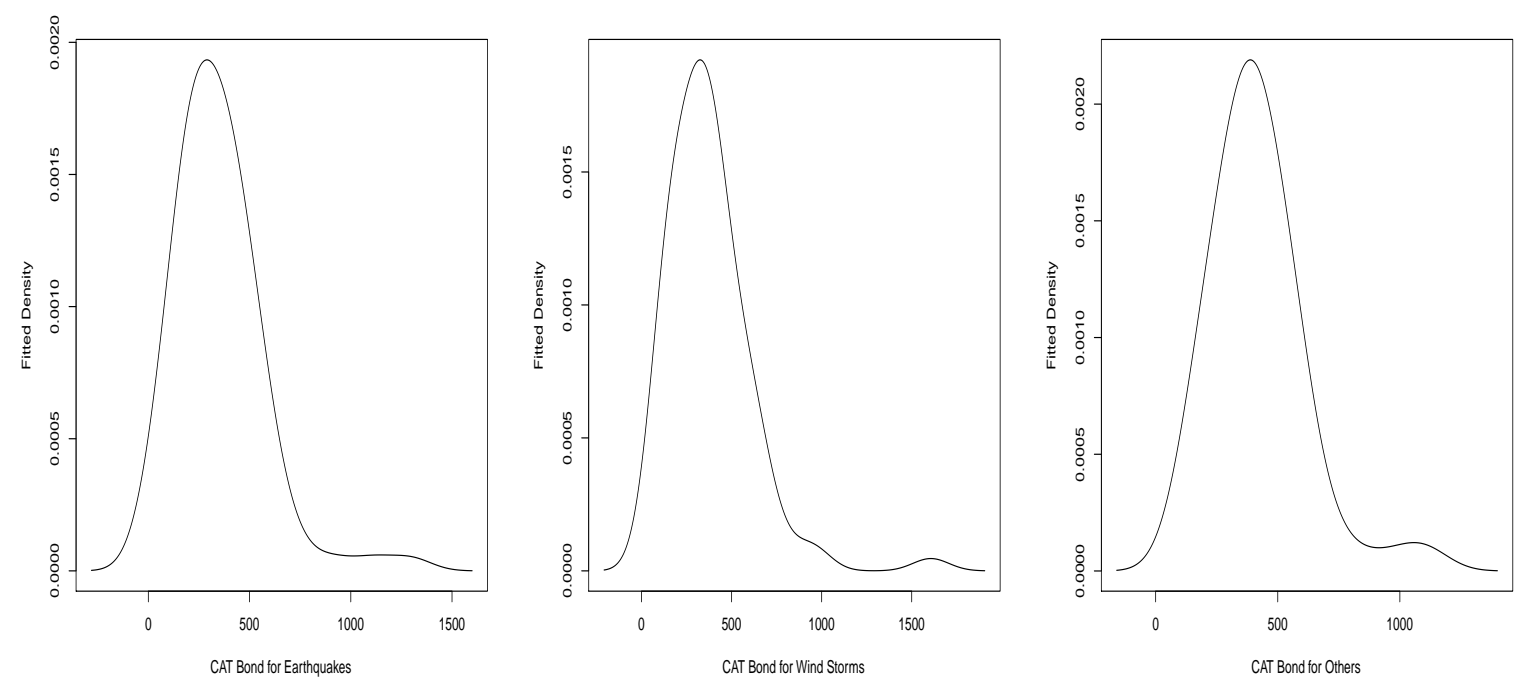

Figure 5: Fitted PDFs of the total catastrophe bond data for earthquakes (left), wind storms (middle) and other disasters (right).

We used Theorem 2.2 to estimate the PDFs of total catastrophe bond due to earthquakes, wind storms and other disasters. The estimated PDFs are shown in Figure 5. These PDFs can assist in the pricing of catastrophe bonds for catastrophes around the world. We can compute the Value at Risk for the different catastrophes which can provide us with information about the different quantile levels of the pricing of the catastrophe bonds. 


\begin{tabular}{llll}
\hline Level & Earthquakes & Wind storms & Others \\
\hline 0.9 & 700.732 & 661.508 & 682.658 \\
0.95 & 849.183 & 806.854 & 780.092 \\
0.99 & 1171.807 & 1139.005 & 968.836 \\
0.999 & 1599.569 & 1601.842 & 1176.331 \\
0.9999 & 2000.824 & 2049.847 & 1328.213 \\
0.99999 & 2382.421 & 2482.279 & 1439.309 \\
0.999999 & 2748.23 & 2904.446 & 1517.236 \\
0.9999999 & 3100.806 & 3310.414 & 1658.039 \\
\hline
\end{tabular}

Table 3: Estimates of Value at Risk for catastrophe bonds.

Table 3 gives Value at Risk estimates of catastrophe bonds for the three types of catastrophes. For quantile levels from 0.9 to 0.99 , the earthquakes give the largest Value at Risk estimates. For quantile levels greater than 0.99 , the wind storms give the largest Value at Risk estimates. For all quantile levels, other catastrophes give the smallest Value at Risk estimates.

\section{References}

[1] Abate, J. and Valkó, P. P. (2004). Multi-precision Laplace transform inversion. International Journal for Numerical Methods in Engineering, 60, 979-993.

[2] Albrecher, H. and Kortschak, D. (2009). On ruin probability and aggregate claim representations for Pareto claim size distributions. Insurance: Mathematics and Economics, 45, $362-373$.

[3] Amari, S. V. and Misra, R. B. (1997). Closed-form expressions for distribution of sum of exponential random variables. IEEE Transactions on Reliability, 46, 519-522.

[4] Bazargan, H., Bahai, H. and Aminzadeh-Gohari, A. (2007). Calculating the return value using a mathematical model of significant wave height. Journal of Marine Science and Technology, 12, 34-42.

[5] Bean, M. A. (2001). Probability: The Science of Uncertainty: With Applications to Investments, Insurance, and Engineering. American Mathematical Society, Providence.

[6] Bonfiglioli, A. and Gancia, G. (2013). Heterogeneity, selection and labor market disparities. Working Paper 734, Barcelona Graduate School of Economics, Spain.

[7] Goovaerts, M. J., Kaas, R., Laeven, R. J. A., Tang, Q. and Vernic, R. (2005). The tail probability of discounted sums of Pareto-like losses in insurance. Scandinavian Actuarial Journal, 6, 446-461.

[8] Hempel, C. G. (2007). Track initialization for multi-static active sonar systems. OCEANS 2007 - Europe, pp. 1-6.

[9] Hitha, N. (1991). Some characterizations of Pareto and related populations. Ph.D. Thesis, Department of Mathematics and Statistics, Cochin University of Science and Technology, Kochi, India. 
[10] Khuong, H. V. and Kong, H. -Y. (2006). General expression for pdf of a sum of independent exponential random variables. IEEE Communications Letters, 10, 159-161.

[11] Klugman, S. A., Panjer, H. H. and Willmot, G. E. (2008). Loss Models, third edition. John Wiley and Sons, Hoboken, New Jersey.

[12] Morales, M. (2005). On an approximation for the surplus process using extreme value theory: Applications in ruin theory and reinsurance pricing. North American Actuarial Journal, 8, 46-66.

[13] Nadarajah, S. (2008). Generalized Pareto models with application to drought data. Environmetrics, 19, 395-408.

[14] Nadarajah, S. and Pogány, T. K. (2013). On the characteristic functions for extreme value distributions. Extremes, 16, 27-38.

[15] Nguyen, Q. H. and Robert, C. (2013). Series expansions for sums of independent Pareto random variables. Under review for publication. A preprint available on-line at http://docs.isfa.fr/labo/2012.16.pdf

[16] Pareto, V. (1964). Cours d'Économie Politique: Nouvelle édition par G. -H. Bousquet et G. Busino, Librairie Droz, Geneva, pp. 299-345.

[17] Pickands, J. (1975). Statistical inference using extreme order statistics. Annals of Statistics, 3, 119-131.

[18] R-forge Distributions Core Team (2008). A guide on probability distributions. http://dutangc.free.fr/pub/prob/probdistr-main.pdf

[19] Ramsay, C. M. (2006). The distribution of sums of certain I.I.D. Pareto variates. Communications in Statistics - Theory and Methods, 35, 395-405.

[20] Ramsay, C. M. (2007). Exact waiting time and queue size distributions for equilibrium M/G/1 queues with Pareto service. Queueing Systems, 57, 147-155.

[21] Ramsay, C. M. (2008). The distribution of sums of I.I.D. Pareto random variables with arbitrary shape parameter. Communications in Statistics-Theory and Methods, 37, 21772184 .

[22] Ramsay, C. M. (2009). The distribution of compound sums of Pareto distributed losses. Scandinavian Actuarial Journal, 27-37.

[23] Wendel, J. G. (1961). The non-absolute convergence of Gil-Pelaez' inversion integral. Annals of Mathematical Statistics, 32, 338-339.

[24] Zaliapin, I. V., Kagan, Y. Y. and Schoenberg, F. P. (2005). Approximating the distribution of Pareto sums. Pure and Applied Geophysics, 162, 1187-1228. 